\title{
Top-down search strategies determine attentional capture in visual search: Behavioral and electrophysiological evidence
}

\author{
Martin Eimer and Monika Kiss \\ Birkbeck College, University of London, London, England
}

\begin{abstract}
To investigate how attentional capture in visual search is affected by generalized top-down search strategies, ERPs and behavioral performance were measured in two experiments where spatially nonpredictive color singleton cues preceded visual search arrays that contained one of two equally likely color singletons. When both singletons served as targets, irrelevant-color singleton cues produced behavioral attentional capture effects and elicited an N2pc component, indicative of a singleton search mode. When responses were required to only one of the two color singletons, the same cues no longer elicited behavioral spatial cuing effects, and the N2pc to these cues was attenuated and delayed, in line with the hypothesis that search was now guided by a feature-specific search strategy. Results demonstrate that the ability of visual singleton stimuli to capture attention is not simply determined by their bottom-up salience, but strongly modulated by top-down task sets.
\end{abstract}

The relative roles of endogenous (voluntary and goaldirected) attention and exogenous (involuntary and stimulus-driven) attention in visual search are still under dispute. There is considerable evidence that perceptually salient but task-irrelevant visual events can capture attention independently of current task sets. For example, reaction times (RTs) to shape singleton targets presented among nontarget shapes (e.g., a diamond target among circle distractors) are delayed when a salient but taskirrelevant color singleton is present in the visual search array, relative to trials without a color distractor (e.g., Theeuwes, 1991). This suggests that color singletons capture attention due to their bottom-up salience, and irrespective of current task set. However, other studies (e.g., Folk \& Remington, 1998; Folk, Remington, \& Johnston, 1992; Folk, Remington, \& Wright, 1994) have suggested that the ability of salient visual events to capture attention is determined by current top-down task sets. In these studies, spatially nonpredictive singleton cues preceded visual search displays, and the presence or absence of attentional capture was inferred from the pattern of spatial cuing effects. Faster RTs in response to visual search targets at cued rather than uncued locations were found only when cue features matched the current task set (e.g., for color singleton cues in blocks where targets were also color singletons), but not when cue features were task irrelevant. These findings support the contingent involuntary orienting hypothesis (Folk et al., 1992), which postulates that attentional capture will be triggered by salient visual stimuli only when these possess attributes that are currently task relevant.
To resolve the dispute between the salience-based bottom-up account of attentional capture advocated by Theeuwes $(1991,1992)$ and the hypothesis that capture is mediated by top-down task set (Folk et al., 1992), Bacon and Egeth (1994) have suggested that attentional capture can be determined either by bottom-up salience or by top-down control, depending on which generalized search strategy is used in a given task context. In search tasks where target items are feature singletons, participants may choose to adopt a singleton detection mode, where they search for any feature discontinuity irrespective of its value. When this search mode is adopted, salient but task-irrelevant visual singletons will capture attention, as observed by Theeuwes (1991). However, this singleton search mode is not available in search tasks where targets are not singletons. Here, participants have to adopt a more finely tuned feature search mode, where they search for specific target-defining features or dimensions. When feature search is used, singletons that do not possess targetdefining features will not capture attention, in line with the observations of Folk et al. (1992). Bacon and Egeth demonstrated that RT costs associated with a salient but task-irrelevant color singleton distractor emerge only when shape targets are themselves singletons, but not when targets are not unique in the shape dimension (e.g., when there are several identical shape targets in the display, or when targets are presented together with additional unique nontarget shapes). In the latter case, participants are forced to abandon singleton search in favor of feature search, and task-irrelevant color singletons no longer capture attention (but see Theeuwes, 2004, for a different interpretation).

M. Eimer, m.eimer@bbk.ac.uk 
According to Bacon and Egeth (1994), the selection of feature versus singleton search is an intentional choice informed by the characteristics of a given visual search task. Because of this emphasis on cognitive control, their proposal is more in line with the top-down account of attentional capture by Folk et al. (1992) than with the alternative salience-based bottom-up view advocated by Theeuwes (1992). Most investigations of these two search modes have used task manipulations designed to force participants into either singleton or feature search. For example, it was demonstrated that when targets cannot be detected by searching for featural discontinuities, irrelevant singletons will not capture attention (e.g., Eimer, Kiss, Press, \& Sauter, 2009; Lamy \& Egeth, 2003; Lamy, Leber, \& Egeth, 2004; Yantis \& Egeth, 1999). ${ }^{1}$ In contrast, when four possible target singleton colors varied unpredictably across trials, so that the feature search mode could not be employed, attentional capture by irrelevant singletons was observed (Lamy \& Egeth, 2003).

Although these results provide evidence that two distinct search modes do in fact exist, they provide little insight into the strategic factors that determine the choice between them. Such factors may be uncovered by studying visual search tasks where both search modes are, in principle, available. In fact, it is not immediately obvious why participants in the studies by Theeuwes (1992) and Bacon and Egeth (1994, Experiment 1) should have chosen a singleton search mode, given that the identity of the shape target (a circle among diamonds) was known and the feature search mode was therefore available. The more precise shape-specific feature search mode would have prevented attentional capture by color singleton distractors and would therefore have improved task performance. Bacon and Egeth have suggested that singleton search mode might impose fewer demands on cognitive control and working memory, which may be sufficient to offset any costs associated with the occasional capture of attention by irrelevant singletons. The singleton search mode may thus be a default strategy adopted whenever possible and replaced by the more demanding feature search mode only under task conditions where the former mode cannot reliably detect visual search targets.

However, findings by Folk and Remington (1998, Experiment 1) challenge the proposed status of singleton search as the default strategy. They used a spatial cuing paradigm similar to the one used by Folk et al. (1992), in which experiment participants searched for targets in a predefined color and target displays were preceded by spatially nonpredictive color singleton cues that either matched or did not match the target color. Spatial cuing effects indicative of attentional capture were observed only when the singleton color cue matched the current target color. Critically, this feature-specific attentional capture effect was observed not only for participants who searched for nonsingleton targets and were therefore forced to adopt a feature search mode, but also for a different group of participants who searched for color singletons. If this group had adopted a singleton search mode, spatial cuing effects should have been observed for target-color cues as well as for irrelevant-color ones. Folk and Reming- ton (1998) suggested that the close temporal proximity of color singleton cues and color targets might have led participants to adopt a feature search mode, although the singleton search strategy was, in principle, available. An alternative possibility is that when observers search for a unique predefined target color, the feature search mode is equally as efficient as, or even more efficient than, singleton search.

To gain more detailed insights into the time course of attentional capture and its modulation by top-down task set, a number of recent studies have measured an event-related brain potential (ERP) component as an electrophysiological marker of capture (e.g., Eimer \& Kiss, 2008; Eimer et al., 2009; Hickey, McDonald, \& Theeuwes, 2006; Lien, Ruthruff, Goodin, \& Remington, 2008). The N2pc component, an enhanced negativity over posterior scalp electrodes contralateral to the side of an attended stimulus elicited between 180 and $300 \mathrm{msec}$ after the onset of a visual search array, is assumed to reflect the attentional selection of candidate target items among distractors in visual search tasks (Luck \& Hillyard, 1994a, 1994b; see also Eimer, 1996; Girelli \& Luck, 1997; Mazza, Turatto, Umiltà, \& Eimer, 2007; Woodman \& Luck, 1999). For example, Eimer and Kiss measured the N2pc in response to spatially uninformative color singleton cues that preceded singleton targets. Behavioral spatial cuing effects were accompanied by an $\mathrm{N} 2 \mathrm{pc}$ in response to red color singleton cues, reflecting rapid attentional capture, when observers had to report the orientation of a uniquely colored red target bar among distractor bars (color task). In contrast, no behavioral cuing effect and no N2pc emerged in response to the same cues when participants had to report the orientation of a gray target bar presented without distractors (onset task), or of a smaller target bar presented among larger distractors (size task). The observation that an N2pc was elicited by physically identical color singleton cues only when their color matched the current task set supports the contingent involuntary attentional capture hypothesis of Folk et al. (1992), and demonstrates that capture is not primarily driven by bottom-up salience. In line with the findings of Folk and Remington (1998), these results also demonstrate that, even though targets were singleton items, participants did not use a singleton search mode but instead opted for a more specific dimension or feature-based search strategy (see Eimer et al., 2009, and Lien et al., 2008, for additional N2pc evidence of taskset-contingent attentional capture in visual search tasks with nonsingleton targets).

The aim of the present study was to combine behavioral and electrophysiological measures of attentional capture to provide further evidence for the existence of distinct generalized search strategies. In particular, we wanted to investigate the conditions that determine whether visual search for color singletons is guided by a singleton or feature search mode, and to study the consequences of adopting one or the other of these two search strategies on behavioral and electrophysiological correlates of attentional capture. We used a cuing procedure similar to the one employed by Folk and Remington (1998). Spatially 


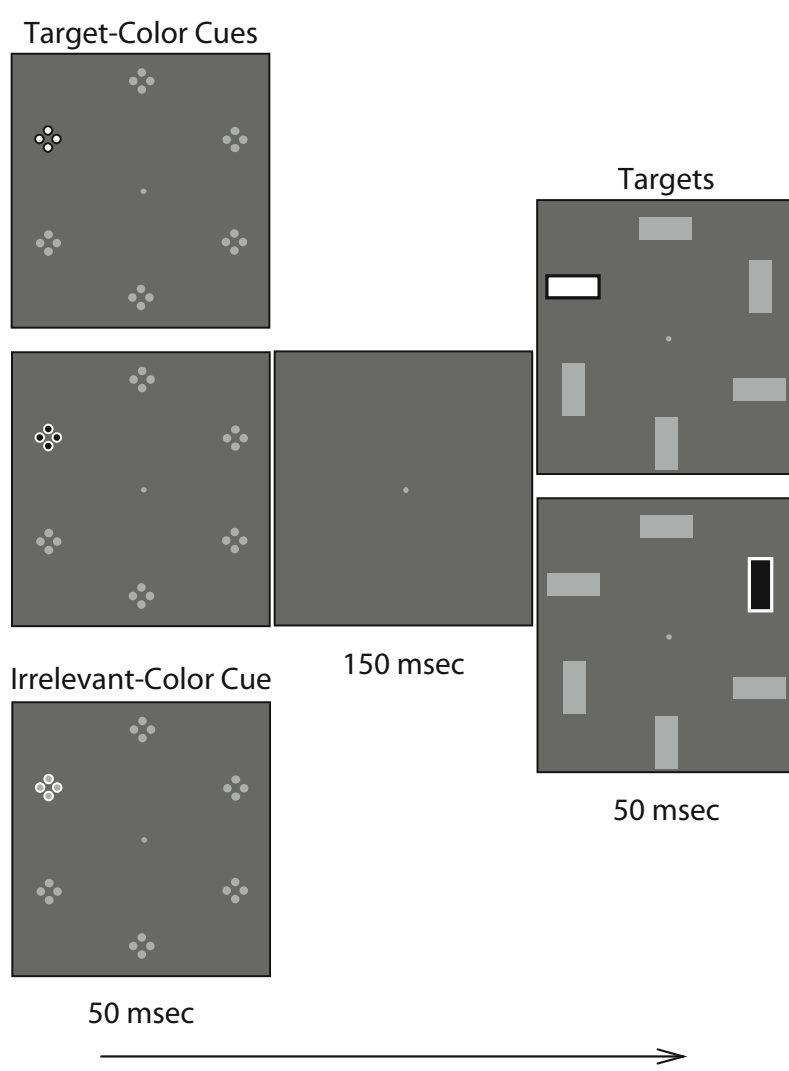

Figure 1. Schematic illustration of trial sequences in Experiment 1 . In all trials, a spatially noninformative cue array was presented for $50 \mathrm{msec}$ and was followed after a blank interval of $150 \mathrm{msec}$ by a search array (50-msec duration) that contained one of two possible color singleton targets among gray items. Cue arrays contained one of the two possible target-color singletons or an irrelevant-color singleton among gray items. Color singletons (red, green, or blue) are shown in black, white, or gray, with different outlines. Stimulation procedures were identical in Experiment 2, but participants now had to respond only to one specific color singleton in the search array, and to refrain from responding to search arrays that contained the nontarget-color singleton.

uninformative color singleton cues preceded visual search displays that contained one color singleton bar among gray distractors (see Figure 1). Three different noninformative color singleton cues (red, green, or blue) were presented with equal probability, and were followed by search displays that contained one of two possible color singleton bars (red or green, red or blue, green or blue, varied across participants). The interval between cue and search array onset was $200 \mathrm{msec}$, in order to ensure that any N2pc triggered by the cues would not overlap with early visual ERP components in response to the subsequent search array.

In Experiment 1, participants had to detect all color singleton bars, regardless of their color, and to indicate their orientation (horizontal or vertical) with a left-hand or right-hand buttonpress. This search task was similar to the one used by Folk and Remington (1998, Experiment 1), who demonstrated feature search in a task with color singleton targets. However, there was one important difference: The color of the target singleton was fixed in the Folk and Remington (1998) study, but two different possible target colors were employed here. The critical question was whether participants would still use a feature search mode or would instead adopt a singleton search mode. If a feature search mode (e.g., "red or green") was used to guide target detection, irrelevant-color cues (e.g., blue singletons in the cue array) should not trigger any attentional capture, because they were not part of the currently active task set. Accordingly-and in contrast to target-color singleton cues - they should not produce behavioral spatial cuing effects indicative of capture and should not trigger an N2pc. Alternatively, if participants adopted a singleton search mode for any color discontinuity regardless of its feature value, behavioral spatial cuing effects and N2pc components should be equally strong for target-color and irrelevant-color singleton cues. Note that the latter prediction is also consistent with the hypothesis that rapid attentional capture is driven exclusively by bottom-up salience rather than top-down task set (Theeuwes, 1992). Experiment 2 was conducted to decide between these two alternative accounts.

\section{EXPERIMENT 1}

\section{Method}

Participants. Seventeen volunteers were paid to participate in this experiment. Three of them were excluded from analyses because of excessive alpha activity, and 2 because of HEOG artifacts (see below for details). The remaining 12 participants ( 5 male, mean age 25 years) had normal or corrected-to-normal vision.

Stimuli and Procedure. Stimuli were presented on a CRT monitor (100-Hz refresh rate) against a black background. On each trial, a cue display was presented for $50 \mathrm{msec}$, and was followed after a $150-\mathrm{msec}$ blank interval by a $50-\mathrm{msec}$ presentation of the search display (see Figure 1). Each cue display consisted of a circular array of six sets of four dots. Each set of dots subtended $0.8^{\circ} \times 0.8^{\circ}$ of visual angle, and was presented at a distance of $4.5^{\circ}$ from a central gray fixation point. Five sets of dots were gray, and one set of dots was a color singleton (red, green, or blue). This color singleton cue appeared randomly and equiprobably at one of the four lateral positions in the left or right hemifield, but never at the top or bottom position. The three singleton cue colors were presented with equal probability and were randomly intermixed within each block. Search displays consisted of circular arrays of six equally spaced horizontal or vertical bars $\left(1.3^{\circ} \times 0.5^{\circ}\right)$ that were presented at the same location as the preceding elements of the cue display ( $4.5^{\circ}$ from fixation). Each search display contained five gray distractor bars and one color singleton target bar. Two different target color singletons (red or green, red or blue, green or blue, counterbalanced across participants) were presented randomly and equiprobably within each block. Target singletons appeared randomly and equiprobably at either of the four lateral positions, but never at the top or bottom position. The orientation of each bar in the search array (horizontal or vertical) was randomly assigned. Gray and colored stimuli in the cue and search displays were all equiluminant $\left(10.3 \mathrm{~cd} / \mathrm{m}^{2}\right)$. The interval between search array offset and the onset of the cue array on the next trial was $1,450 \mathrm{msec}$

Participants' task was to search for the color singleton target bar and report its orientation (horizontal or vertical) by pressing one of two vertically arranged response keys with the left or right hand. Hand-to-key assignment was reversed after half of the blocks. Prior to the start of the experiment, participants were reminded that target singletons could be in one of two colors. They were instructed to respond as fast and accurately as possible to search arrays while maintaining central fixation. The experiment consisted of 10 blocks with 96 trials per block. Because the locations of color singleton cues and targets were randomly assigned on each trial, cue arrays 
were spatially uninformative, and participants were instructed to ignore them.

EEG data recording and analysis. The EEG was DC-recorded from 23 scalp electrodes mounted in an elastic cap at standard positions of the extended 10/20 system at sites Fpz, Fz, F3, F4, F7, F8, FC5, FC6, Cz, C3, C4, T7, T8, CP5, CP6, Pz, P3, P4, P7, P8, PO7, $\mathrm{PO} 8$, and $\mathrm{Oz}$. The continuous EEG was sampled at a rate of $500 \mathrm{~Hz}$ and digitally low-pass filtered with a $40-\mathrm{Hz}$ Butterworth filter. No further filters were applied after EEG acquisition. All electrodes were online referenced to the left earlobe and were rereferenced offline to the average of both earlobes. Only trials with correct responses to targets were analyzed. These trials were segmented from $100 \mathrm{msec}$ before the onset of the cue display to $500 \mathrm{msec}$ after cue onset. Trials with artifacts (HEOG exceeding $\pm 30 \mu \mathrm{V} ; \mathrm{Fpz} \pm 60 \mu \mathrm{V}$; all other electrodes $\pm 80 \mu \mathrm{V}$ ) were removed from the analysis. Two participants were excluded because their averaged HEOG exceeded $\pm 3 \mu \mathrm{V}$, indicating a residual tendency to move their gaze toward the side where color singletons were presented.

EEG was averaged for all combinations of cue type (target-color cue, collapsed across both target colors vs. irrelevant-color cue) and cue side (left vs. right visual field), collapsed across all four possible color singleton target locations. The N2pc to color singleton cues was quantified on the basis of mean amplitudes obtained in a 180- to 280-msec time window after cue onset at lateral posterior electrodes PO7 and PO8. N2pc mean amplitudes were analyzed in a repeated measures ANOVA with the factors cue type (target color vs. irrelevant color), cue side, and contralaterality (electrode contralateral vs. ipsilateral to the visual field of the color singleton cue). $\mathrm{N} 2 \mathrm{pc}$ onset latencies in response to target-color and irrelevant-color cues were compared with the jackknife-based method described by Miller, Patterson, and Ulrich (1998) on the basis of difference waveforms obtained by subtracting ipsilateral from contralateral ERPs. This jackknife procedure estimates onset latencies from grand averages computed for subsamples of participants by successively excluding 1 participant from the original sample. The onset was defined as the first time point at which the voltage in the negativegoing difference waveform in a subsample exceeded $40 \%$ of the N2pc peak amplitude (see Eimer et al., 2009, for identical procedures). For the latency comparison, the $t$ value was corrected according to the formula given by Miller et al. (1998).

\section{Results}

Behavioral results. Trials with premature responses (RTs faster than $200 \mathrm{msec}$ ) or absent responses were rare (fewer than $0.1 \%$ of all trials) and were excluded from analysis. Figure 2 (left panel) shows the pattern of behavioral performance on trials in which color singleton cues and targets appeared at the same location and at different locations, separately for trials with target-color and irrelevant-color singleton cues. The expected spatial cuing effect on RTs (faster responses on same-location than on different-location trials) was obtained for target-color cues. Importantly, a spatial cuing effect was also present on trials where irrelevant-color cues were presented.

This pattern was confirmed by statistical analysis of the RT data for the factors cue-target location (same vs. different) and cue type (target-color vs. irrelevant-color cue). A main effect of cue-target location $[F(1,11)=68.7, p<$ $.001]$, reflecting faster RTs for same-location trials, was accompanied by an interaction between cue type and cuetarget location $[F(1,11)=5.0, p<.05]$, indicating that this spatial cuing effect was larger with target-color cues than with irrelevant-color cues. Follow-up analyses conducted separately for both cue types confirmed that spatial

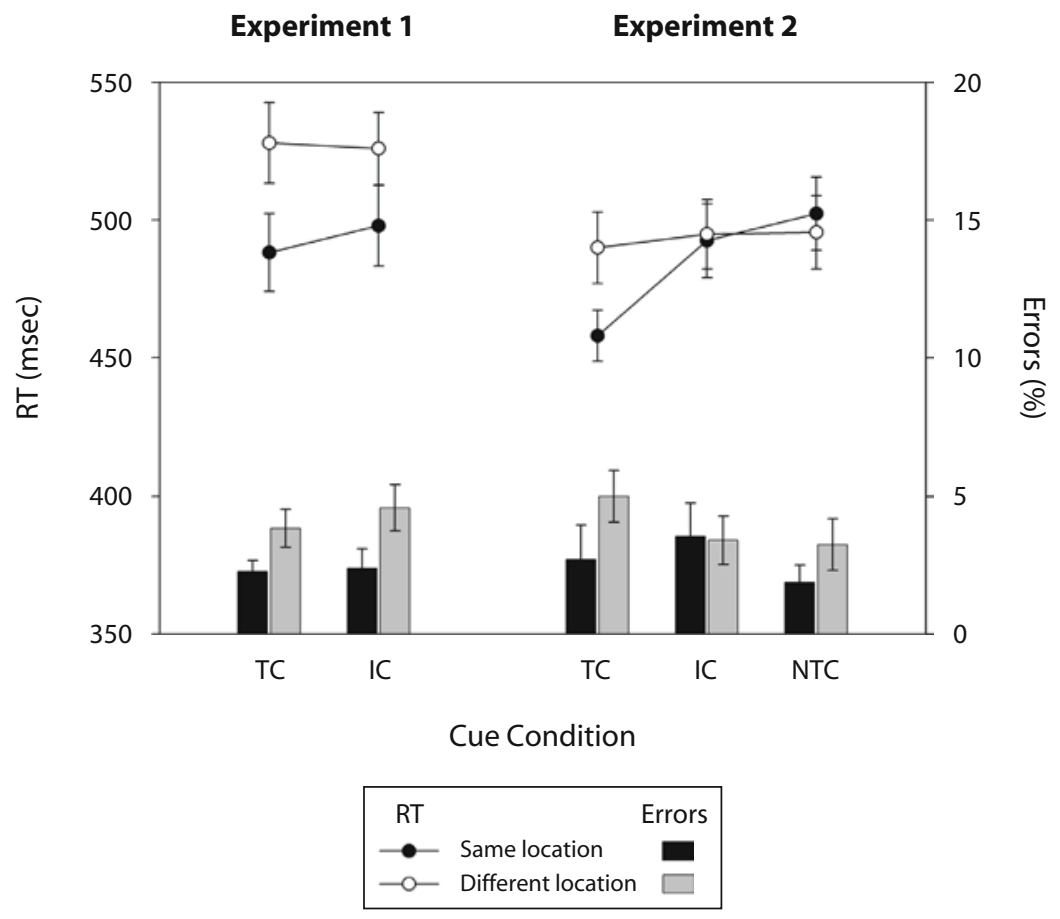

Figure 2. Response times (RTs; line graphs) and error rates (bar graphs) in Experiments 1 and 2. Results for trials where color singleton cues and targets were presented at the same or at different locations are shown separately for each singleton cue type (TC, target-color cue; IC, irrelevant-color cue; NTC, nontarget-color cue, for Experiment 2 only). 
cuing effects were reliably present not just for target-color cues [488 vs. $528 \mathrm{msec} ; t(11)=10.5, p<.001]$, but also for irrelevant-color cues [498 vs. $526 \mathrm{msec} ; t(11)=4.9$, $p<.001]$. A similar pattern was observed for response errors (incorrect keypresses), which were more frequent on different-location trials than on same-location trials [main effect of cue-target location: $F(1,11)=9.3, p<$ $.05]$. There was no interaction between cue type and cuetarget location for error rates $[F(1,11)=1.7, p=.222]$, indicating that spatial cuing effects on accuracy were comparable for target-color and irrelevant-color cues (see Figure 2, left panel).

N2pc. Figure 3 shows ERPs elicited at electrodes PO7/8 contralateral and ipsilateral to the position of a color singleton cue in the 300-msec interval after cue array onset, together with the resulting contralateral minus ipsilateral difference waveforms and N2pc scalp distribution maps, separately for trials with target-color and irrelevant-color cues. An N2pc was triggered not only in response to targetcolor cues, but also for irrelevant-color cues, although $\mathrm{N} 2 \mathrm{pc}$ onset appears slightly delayed for these cues.

The analysis of ERP mean amplitudes in the 180- to 280-msec time window following cue array onset found a main effect of contralaterality $[F(1,11)=20.5, p<.001]$, reflecting the presence of an N2pc to color singleton cues. The interaction between cue type and contralaterality did not reach significance $[F(1,11)=2.7, p=.127]$, indicating that N2pc amplitudes did not differ reliably between target-color and irrelevant-color cues. Separate analyses conducted for target-color and irrelevant-color cues confirmed the presence of N2pc components for both cue types $[F(1,11)=20.9$ and 17.9 , both $p$ s $<.001]$, for target-color and irrelevant-color cues, respectively. A jackknife-based analysis of onset latencies for N2pcs to target-color and irrelevant-color cues showed that the latency difference illustrated in Figure 3 did not reach sta-

\section{Target-Color Cue}

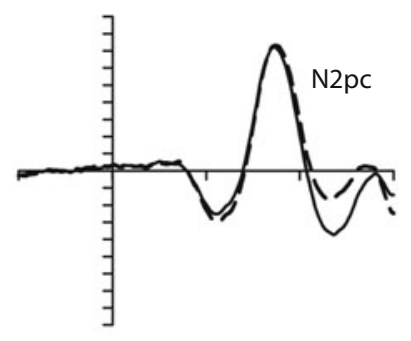

Irrelevant-Color Cue
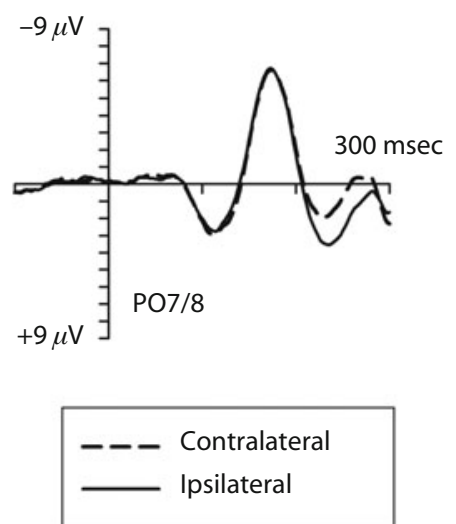

\section{Difference Waves}

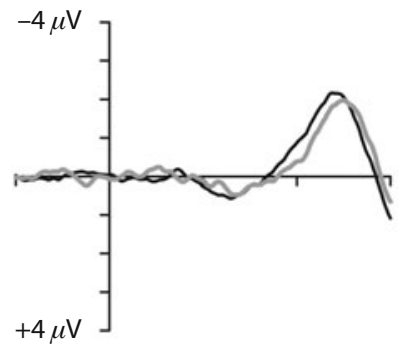

Target-color cue Irrelevant-color cue

Difference Maps (180-280 msec)

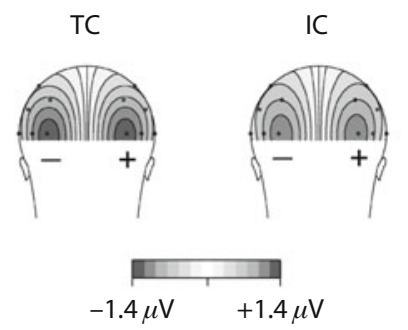

Figure 3. Left panel: ERPs elicited in Experiment 1 in the 300-msec interval after cue onset in response to target-color and irrelevant-color singleton cues at posterior electrode sites PO7/8 contralateral (dashed lines) and ipsilateral (solid lines) to the visual hemifield where the color singleton cue was presented. Each tick on the $x$-and $y$-axes represents steps of $100 \mathrm{msec}$ and $1 \mu \mathrm{V}$, respectively. Top right panel: Difference waveforms obtained by subtracting ipsilateral from contralateral ERPs in response to target-color singleton cues (black line) and irrelevant-color singleton cues (gray line). Bottom right panels: Topographical maps (back views) of $\mathrm{N2pc}$ scalp distributions in the 180- to 280-msec postcue interval for target-color (TC) and irrelevant-color (IC) cue arrays. Maps were constructed by spherical spline interpolation (see Perrin, Pernier, Bertrand, \& Echallier, 1989) after mirroring the ipsilateral-contralateral difference waveforms to obtain symmetrical voltage values for both hemispheres. The N2pc appears as negative voltage $(-)$ over the left hemisphere and as positive voltage $(+)$ over the right hemisphere. 
tistical significance [201 vs. $215 \mathrm{msec} ; t_{\mathrm{c}}(11)=1.44, p=$ .08 , one-tailed].

\section{Discussion}

When participants searched for color singleton bars that could have one of two colors, both behavioral and ERP measures demonstrated that attention was captured not only by color singleton cues that matched one of these two target colors, but also by irrelevant-color cues. This strongly suggests that participants had adopted a singleton search mode. As expected, behavioral spatial cuing effects indicative of attentional capture were observed on trials where target-color cues were presented. These cues also triggered pronounced N2pc components, confirming findings from previous ERP studies (Eimer \& Kiss, 2008; Eimer et al., 2009; Lien et al., 2008) and emphasizing the fact that target-color cues captured attention. The critical question was whether similar behavioral and electrophysiological effects would also be observed for irrelevant-color cues. This turned out to be the case, and strongly suggests that color singleton cues captured attention in spite of their color being nominally task irrelevant. Behavioral spatial cuing effects were slightly but reliably larger for target-color cues, and there was also a nonsignificant trend toward an N2pc onset delay for irrelevantcolor cues. Both observations suggest that a color-specific top-down task set may have had a small modulating effect on attentional capture by color singleton cues. However, the main finding of Experiment 1 was that both behavioral and ERP measures provided convergent evidence that color singleton cues captured attention even when they did not match one of the two current target colors, in line with the assumption that participants chose to adopt a singleton search mode.

It has previously been suggested that the capacity of target-color singleton cues to capture attention is modulated by intertrial priming, since it is more pronounced on trials where the color of a cue matches the target color on the preceding trial (Folk \& Remington, 2008). There was little evidence for such intertrial priming effects in Experiment 1: Even though spatial cuing effects were numerically larger when the color of the singleton cue matched the color of the preceding target relative to nonmatching target-color cues ( $43 \mathrm{vs.} 36 \mathrm{msec}$ ), this difference was not reliable $[F(1,11)=1.3]$. Similarly, N2pc amplitudes did not differ between matching and nonmatching target-color cues $[F(1,11)=1.1]$.

In a previous behavioral study where observers searched for color singleton targets, Folk and Remington (1998) found that attentional capture was triggered only by color singleton cues that matched the current target color, but not by irrelevant-color singleton cues, suggesting that search was guided by a color-specific feature search strategy. The difference between these findings and the present results suggests that the choice between singleton and feature search modes is optional, and is determined by the tendency to minimize the demands on attentional control and working memory during task execution. In the Folk and Remington (1998) study, target color was fixed, whereas participants in Experiment 1 had to search for one of two possible color singleton targets. In the latter case, they may have opted for the lower-cost singleton search mode, because the alternative feature search mode would have required the active maintenance of a task set that included two target colors. In contrast, when target color is constant, feature search may be equally or even less demanding than singleton search, and will therefore become the preferred option.

There is, however, an alternative, simpler interpretation of the pattern of results observed in Experiment 1: Instead of assuming that participants adopted a singleton search mode because this was the more efficient strategy, attentional capture by salient but irrelevant color singleton cues could have been triggered in a bottom-up fashion, independently of current task sets and search strategies (e.g., Theeuwes, 1992). Experiment 2 was conducted to decide between these two alternatives.

\section{EXPERIMENT 2}

In Experiment 1, nominally task-irrelevant color singleton cues captured attention. This may reflect either salience-driven bottom-up capture or the fact that participants chose to adopt a singleton search strategy. To decide between these two alternatives, Experiment 2 employed exactly the same visual stimuli and procedures as did Experiment 1, except that task instructions were changed. Whereas both color singleton targets were responserelevant in Experiment 1, participants now responded to the orientation of bars in one predefined target color, but had to refrain from responding on trials where a singleton bar in the other color was presented. With these new instructions, the target or nontarget status of a color singleton in a search array was defined by its color. Therefore, a feature-unspecific singleton search strategy could no longer be employed, and participants instead had to adopt a color-specific search mode (see Folk \& Remington, 2008, Experiment 2, for similar procedures).

As in Experiment 1, an N2pc and behavioral spatial cuing effects indicative of attentional capture were predicted in response to target-color singleton cues. The critical question was whether irrelevant-color singleton cues (i.e., cues that matched the color of neither target nor nontarget singletons in the search arrays) would also still capture attention. If attentional capture by these singleton cues is a salience-driven bottom-up phenomenon independent of task sets and top-down search strategies, the behavioral and ERP correlates of attentional capture found for irrelevant-color cues in Experiment 1 should again be observed in Experiment 2, since low-level stimulus features remained unchanged. Theeuwes, Atchley, and Kramer (2000) have argued that, even though salient singleton stimuli always capture attention in a bottomup fashion, attention is rapidly disengaged from stimuli that have no task-set-relevant attributes. Such a rapid disengagement from singleton cues may prevent the emergence of behavioral spatial cuing effects in response to subsequent target arrays. If this was the case, no such cuing effects would be observed for irrelevant-color cues in Experiment 2, but an early N2pc component indica- 
tive of rapid attentional capture should still be triggered by these cues. In contrast, if attentional capture by color singletons is determined by generalized top-down search strategies, behavioral and electrophysiological capture effects should be absent or strongly attenuated for irrelevant-color cues as compared with target-color cues, since task instructions in Experiment 2 required a colorspecific feature search strategy.

\section{Method}

Participants. Fifteen paid volunteers participated in Experiment 2. Two were removed from the analyses because of HEOG artifacts, and 1 was excluded because of very slow responses (mean RTs in all conditions were $2.5 \mathrm{SDs}$ above the sample means). The remaining 12 participants ( 4 male, mean age 27.7 years) had normal or corrected-to-normal vision.

Stimuli and Procedure. Cue and search displays and presentation procedures were identical to those in Experiment 1, except that one of the two possible color singletons in the search array was now designated as a nontarget stimulus, to which no response was required.

EEG data recording and analysis. Two participants were excluded because their averaged HEOG exceeded $\pm 3 \mu \mathrm{V}$. EEG recording and analyses were identical to those in Experiment 1, except that ERPs were now computed separately for three different color singleton cue types (target-color cue, irrelevant-color cue, nontarget-color cue), and the factor cue type now had three levels. Greenhouse-Geisser corrections for nonsphericity were applied where appropriate.

\section{Results}

Behavioral results. Figure 2 (right panel) shows the pattern of behavioral performance on trials where color singleton cues and targets appeared at the same or at a different location, separately for trials with target-color cues, irrelevant-color cues, and nontarget-color cues. RT data include only correct responses on trials where search arrays contained a target-color singleton. As in Experiment 1 , responses were faster on same-location than on different-location trials on trials where target-color cues were presented. In marked contrast to Experiment 1, there was no longer any spatial cuing effect in response to irrelevant-color cues. For trials with nontarget-color cues, RTs were even slightly faster on different-location than on same-location trials.

Statistical analyses revealed a main effect of cue type on $\operatorname{RT}[F(2,22)=18.7, p<.001]$, with faster RTs on trials with target-color cues than on trials with irrelevant-color or nontarget-color cues. As can be seen in Figure 2 (right panel), this effect is due to the fact that RTs were much faster on trials where targets were preceded by targetcolor cues at the same location than on all other types of trials. Accordingly, the interaction between cue type and cue-target location was highly significant $[F(2,22)=$ $13.4, p<.001]$. To further explore this interaction, RTs on same-location and different-location trials were compared separately for each of the three cue types. For target-color cues, RTs were reliably faster on same-location than on different-location trials [458 vs. $490 \mathrm{msec} ; t(11)=4.1$, $p<.01]$. No such spatial cuing effect was present for irrelevant-color cues (492 vs. $495 \mathrm{msec}$ for same-location and different-location trials; $t<1$ ). Following nontargetcolor cues, RTs were numerically slower on same-location than on different-location trials, but this difference was not reliable [502 vs. $495 \mathrm{msec} ; t(11)<1.3, p=.217$ ].

Error rates were generally low. False alarms were observed on fewer than $0.1 \%$ of all nontarget-color cue trials. Incorrect responses were recorded on $3.2 \%$ of all target trials, and errors were less frequent on same-location than on different-location trials $[2.7 \%$ vs. $3.9 \% ; F(1,11)=8.3$, $p<.05]$. There was no interaction between cue-target location and cue type for error rates $(F<1.1)$.

N2pc. Figure 4 shows ERPs elicited at electrodes PO7/8 contralateral and ipsilateral to the position of a color singleton cue in the $300-\mathrm{msec}$ interval after cue array onset, together with the resulting contralateral minus ipsilateral difference waveforms and N2pc scalp distribution maps, separately for trials with target-color cues, irrelevantcolor cues, and nontarget-color cues. A strong N2pc component was elicited in response to target-color cues, as in Experiment 1. In contrast, a delayed and strongly attenuated N2pc was triggered by both irrelevant-color cues and nontarget-color cues.

The analysis of ERP mean amplitudes in the 180- to 280-msec time window following cue array onset found a main effect of contralaterality $[F(1,11)=24.3, p<.001]$, confirming the presence of an N2pc to color singleton cues. Importantly, there was now also a significant interaction between cue type and contralaterality $[F(2,22)=$ $18.9, p<.001]$. To investigate this interaction, separate analyses were conducted for pairwise combinations of two cue conditions, with cue type now a two-level factor. When ERPs to target-color and irrelevant-color cues were analyzed together, a reliable cue type $\times$ contralaterality interaction $[F(1,11)=22.0, p<.001]$ demonstrated that in contrast to the results obtained in Experiment 1, the N2pc was now larger in response to target-color cues. Likewise, when ERPs to target-color and nontarget-color cues were analyzed together, a cue type $\times$ contralaterality interaction $[F(1,11)=21.7, p<.001]$ confirmed larger $\mathrm{N} 2 \mathrm{pc}$ amplitudes on target-color trials. In contrast, no difference in N2pc amplitudes was found between irrelevantcolor and nontarget-color cues $[F(1,11)=1.8, p=.21]$. Analyses conducted separately for each type of cue found reliable effects of contralaterality for all three cue types [all $F \mathrm{~s}(1,11)>9.3$, all $p \mathrm{~s}<.02$ ], demonstrating that even though the N2pc was strongly attenuated in response to irrelevant-color and nontarget-color cues, it was still reliably present for these two types of singleton cues.

Jackknife-based N2pc latency analyses (with N2pc onset defined as $40 \%$ of peak amplitude, as in Experiment 1) confirmed that the N2pc started earlier in response to target-color cues relative to irrelevant-color cues [185 vs. $198 \mathrm{msec} ; t_{\mathrm{c}}(11)=1.9, p<.05$, one-tailed] and relative to nontarget-color cue trials $\left[207 \mathrm{msec} ; t_{\mathrm{c}}(11)=\right.$ $2.77, p<.01$, one-tailed]. The 9-msec delay of the N2pc in response to nontarget-color cues as compared with irrelevant-color cues did not reach significance $\left[t_{c}(11)=\right.$ $1.5, p=.08$, one-tailed].

To demonstrate that the change in task instructions between Experiments 1 and 2 resulted in a reliable attenuation of N2pc amplitudes for irrelevant-color singleton cues relative to target-color cues, an additional analysis 


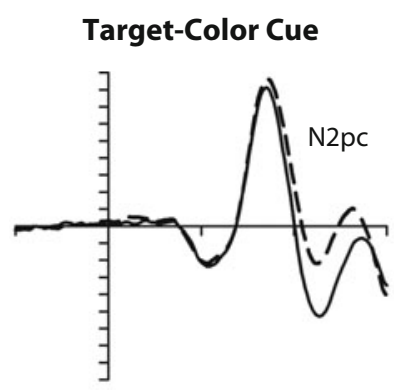

\section{Irrelevant-Color Cue}

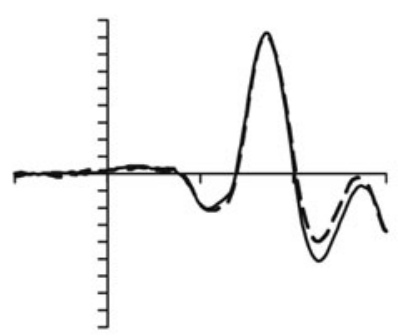

Nontarget-Color Cue

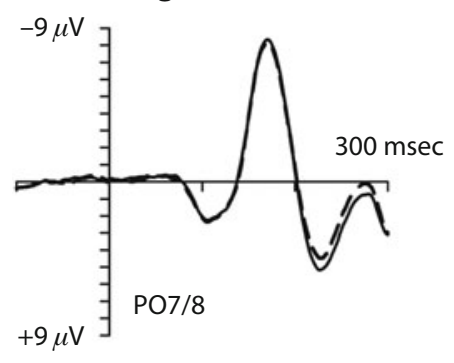

$$
\begin{array}{ll}
-\boldsymbol{-} \text { Contralateral } \\
\longrightarrow \text { Ipsilateral }
\end{array}
$$

Difference Waves
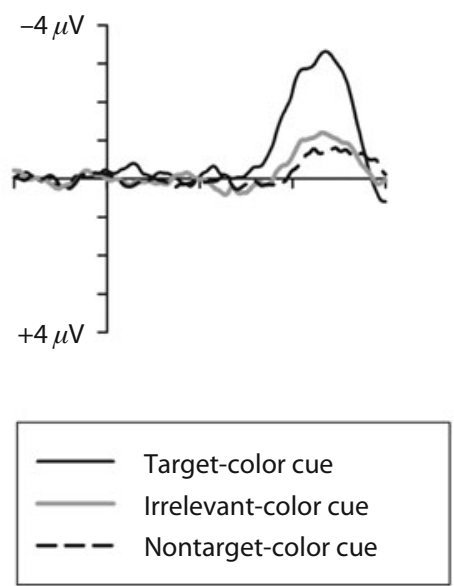

Difference Maps (180-280 msec)

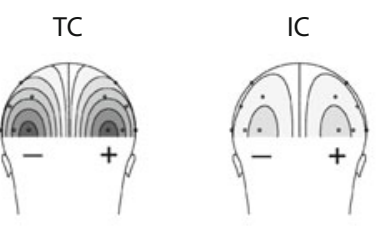

NTC

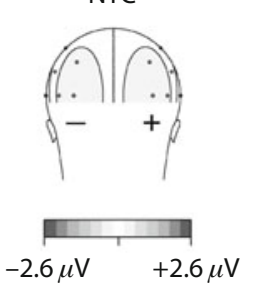

Figure 4. Left panel: ERPs elicited in Experiment 2 in the 300-msec interval after cue onset in response to target-color, irrelevant-color, and nontarget-color singleton cues at posterior electrode sites PO7/8 contralateral (dashed lines) and ipsilateral (solid lines) to the visual hemifield of the color singleton cue. Top right panel: Difference waveforms obtained by subtracting ipsilateral from contralateral ERPs, shown separately for each of the three cue types. Bottom right panels: Topographical maps (back views) of N2pc scalp distribution in the 180- to 280-msec postcue interval for target-color (TC), irrelevant-color (IC), and nontarget-color (NTC) cue arrays.

compared N2pc amplitudes in response to these two types of cues across the two experiments (excluding the nontarget-color cue trials of Experiment 2). A highly significant three-way interaction between contralaterality, cue type, and experiment was obtained $[F(1,22)=14.3$, $p<.001]$, confirming that $\mathrm{N} 2 \mathrm{pc}$ amplitude differences between target-color and irrelevant-color singleton cues were much more pronounced in Experiment 2.

An additional median-split analysis was conducted to further explore the presence of N2pc components in response to irrelevant-color and nontarget-color cues in Experiment 2. If these components reflected the capture of attention by singleton cues that did not match the current task set on a subset of trials, this might be revealed by sorting trials on the basis of RTs to subsequent visual search targets. Figure 5 shows ERPs elicited at PO7/8 contralateral and ipsilateral to the position of irrelevant-color or nontarget-color singleton cues $^{2}$ on same-location and different-location trials, separately for trials where RTs to subsequent visual search target were faster or slower than the median RT (mean RTs: 444 and $552 \mathrm{msec}$ for same-location fast and slow trials, 442 and $549 \mathrm{msec}$ for different-location fast and slow trials). Attentional capture by irrelevant-color or nontarget-color cues should result in 

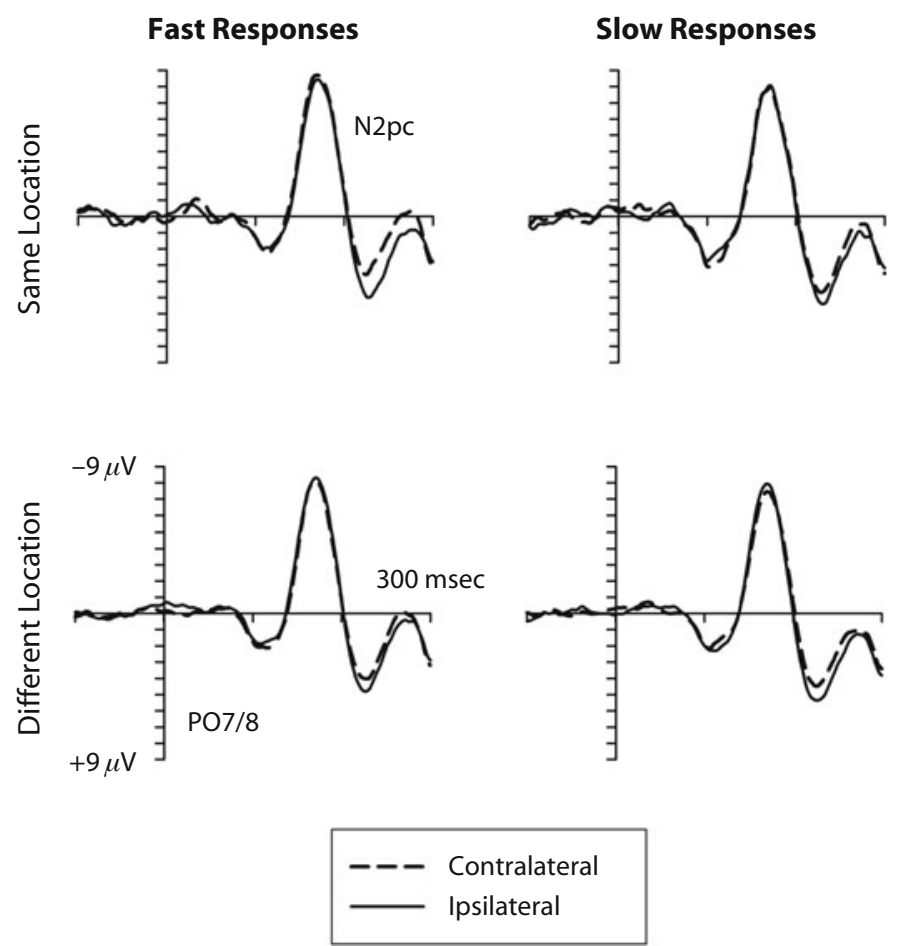

Figure 5. ERPs elicited in Experiment 2 in the 300-msec interval after the onset of irrelevant-color or nontarget-color cues at posterior electrode sites PO7/8 contralateral (dashed lines) and ipsilateral (solid lines) to the visual hemifield of the cue. Data are collapsed across trials with irrelevant-color and nontarget-color cues. ERPs are shown separately for same-location trials (top panels) and different-location trials (bottom panels) as a function of response speed quantified on the basis of RT median splits (fast responses, left panels; slow responses, right panels).

fast RTs on same-location trials where search targets are presented at cued locations, and in slow RTs on differentlocation trials where targets appear at uncued locations. In line with this prediction, N2pc amplitudes were larger on same-location fast and different-location slow trials (top left and bottom right panels in Figure 5) than on samelocation slow and different-location fast trials. An analysis of N2pc mean amplitudes in the 180 - to 280 -msec postcue time interval revealed a reliable three-way interaction between cue-target location (same vs. different), response speed (fast vs. slow RTs to targets), and contralaterality $[F(1,11)=12.2, p<.01]$, and follow-up analyses confirmed that $\mathrm{N} 2 \mathrm{pc}$ components were present on samelocation fast trials $[F(1,11)=20.4, p<.001]$ and on different-location slow trials $[F(1,11)=7.5, p<.02]$, whereas no reliable N2pc was obtained on same-location slow and different-location fast trials [both $F \mathrm{~s}(1,11)<$ 2.8 , both $p \mathrm{~s}>.122]$.

A final set of analyses tested whether the N2pc in response to irrelevant-color and nontarget-color cues on same-location fast trials (Figure 5, top left panel) differed from the N2pc to target-color cues. For ERP mean amplitudes, a significant interaction between cue type and contralaterality $[F(1,11)=14.3, p<.01]$ demonstrated that the N2pc was larger in response to target-color cues. Likewise, N2pc onset was reliably earlier following a target-color cue than in response to irrelevant-color and nontarget-color cues on same-location fast trials [185 vs. $211 \mathrm{msec} ; t_{\mathrm{c}}(11)=3.74, p<.002$, two-tailed].

\section{Discussion}

The aim of Experiment 2 was to determine whether the attentional capture effects observed for irrelevant-color singleton cues in Experiment 1 were due to the fact that participants chose to adopt a color-unspecific singleton search strategy or were triggered in a bottom-up saliencedriven fashion. The results of Experiment 2 support the first hypothesis. Target-color singleton cues again captured attention, as reflected by behavioral spatial cuing effects and the presence of an N2pc. In marked contrast to Experiment 1, no behavioral spatial cuing effects were triggered by irrelevant-color singleton cues, and the N2pc to these cues was strongly attenuated and delayed (see Figure 4). These observations are consistent with the hypothesis that a color-specific feature search mode was adopted in Experiment 2, because this was necessitated by the new task instructions. They provide no support for the view that attentional capture by nontarget singletons is a bottom-up phenomenon triggered regardless of current task sets (e.g., Theeuwes, 1992). The absence of behavioral cuing effects for irrelevant-color singleton cues might be accounted for by the "rapid capture followed by disengagement" hypoth- 
esis suggested by Theeuwes et al. (2000), but the N2pc results obtained in Experiment 2 are inconsistent with this view. Rapid attentional capture by both target-color and irrelevant-color singleton cues should have been reflected in a similar early $\mathrm{N} 2 \mathrm{pc}$ for both types of cues, whereas a subsequent attentional disengagement for task-irrelevant nontarget color cues might have resulted in a shorter-lived $\mathrm{N} 2 \mathrm{pc}$ component. The observation that the N2pc to these cues was delayed and strongly attenuated relative to the $\mathrm{N} 2 \mathrm{pc}$ to target-color cues is clearly inconsistent with these predictions.

The results observed on trials with nontarget-color singleton cues were very similar to those obtained in response to irrelevant-color cues. There were no behavioral spatial cuing effects, and the attenuation of the N2pc component relative to target-color cues was the same for both cue types. This is interesting, because search arrays with a nontarget-color singleton were presented on half of all trials. Because the nontarget color was task relevant in this respect, it could have been part of the currently active task set, and thus might have captured attention in a similar fashion as the target color. Instead, the results of Experiment 2 suggest that participants adopted a task set that specified only the current target color and treated the nontarget color as task irrelevant. This is in line with the assumption that participants will choose a task set that minimizes the demands on working memory, and ideally includes just a single task-relevant feature.

As in Experiment 1, there was little evidence that attentional capture effects in response to target-color cues were affected by intertrial priming (as suggested by Folk \& Remington, 2008). Spatial cuing effects on trials where target-color cues were preceded by search arrays that contained a target or a nontarget singleton were almost identical ( $32 \mathrm{vs} .31 \mathrm{msec}$ ). There was also no effect of the preceding search array on spatial cuing effects triggered by irrelevant-color cues [both $F \mathrm{~s}(1,11)<1]$. However, a small but reliable inverted spatial cuing effect was found when nontarget-color cues were preceded by search arrays with a nontarget singleton [520 and $505 \mathrm{msec}$ for samelocation vs. different-location trials; $t(11)=3.1, p<.02]$, suggesting that encountering search arrays that included a nontarget-color singleton resulted in some locationspecific inhibition in response to a nontarget-color singleton cue on the subsequent trial.

Even though N2pc components to irrelevant-color and nontarget-color singleton cues were strongly attenuated and delayed relative to the N2pc to target-color cues in Experiment 2, they were nevertheless reliably present, suggesting that these cues attracted attention to some degree, on at least some trials. This was confirmed by the additional RT median split analysis, which found reliable $\mathrm{N} 2 \mathrm{pc}$ components for same-location trials with fast responses to subsequent targets and different-location trials with slow responses, but not for same-location slow and different-location fast trials (Figure 5). This is exactly what would be expected if irrelevant-color and nontarget-color cues had captured attention on some trials, since this type of attentional capture would result in fast RTs to targets at cued locations and slow RTs to targets at uncued loca- tions. What remains puzzling is that, in spite of this apparent occasional capture of attention by irrelevant-color and nontarget-color cues (as revealed by the N2pc), no systematic behavioral spatial cuing effect was observed for either type of cue across all trials. A possible explanation is that positive cuing effects triggered on capture trials were offset by inverted cuing effects (i.e., faster RTs on different-location relative to same-location trials) of similar magnitude on no-capture trials (i.e., on trials where no reliable N2pc was elicited). This issue will be further considered in the General Discussion. It should also be noted that even on capture trials, the N2pc to irrelevant-color and nontarget-color cues was smaller and delayed relative to the N2pc observed for target-color cues, which further emphasizes the strong effects of a color-specific task set on attentional capture in Experiment 2.

\section{GENERAL DISCUSSION}

The present study combined behavioral and ERP measures of attentional capture in visual search to provide new evidence for the existence of two generalized top-down search strategies (feature vs. singleton search modes; Bacon \& Egeth, 1994). In Experiment 1, where participants searched for two equally likely color singleton targets, both target-color and irrelevant-color singleton cues captured attention, as reflected by behavioral spatial cuing effects and the presence of N2pc components in response to both types of cues. The observation that irrelevantcolor singletons captured attention suggests that participants adopted a singleton search strategy but could also reflect a bottom-up effect that was driven by salience alone. In Experiment 2, physically identical stimulation procedures were employed, except that participants now had to respond to only one color singleton target, but to refrain from responding when a search array contained the other color singleton. Under these conditions, target-color cues triggered behavioral spatial cuing effects, whereas no such effects were observed for irrelevant-color and nontarget-color cues. Furthermore, the N2pc to these cues was strongly attenuated and delayed relative to the $\mathrm{N} 2 \mathrm{pc}$ triggered by target-color cues. Because physical stimulus parameters were identical in both experiments, this marked difference in the ability of irrelevant-color cues to capture attention across experiments cannot be due to low-level visual factors such as salience (e.g., Theeuwes, 1992). Instead, it is likely to reflect the difference in generalized top-down search strategies, with target detection guided by a singleton search mode in Experiment 1, and by a feature search strategy in Experiment 2 .

In Experiment 1, participants could in principle have chosen a disjunctive color-specific task set (e.g., "red or green"), but the behavioral and ERP data demonstrated that they opted instead for a singleton search mode ("any color singleton"). In Experiment 2, the color-specific task set could, in principle, have included both target and nontarget colors (e.g., "response to red, no response to green"), but results demonstrated that search was instead guided by a task set that specified only the target color, whereas irrelevant-color singletons were treated as task-irrelevant 
distractors. Both findings suggest that the choice of a specific search strategy is determined by the general aim of minimizing working memory load. Adopting a disjunctive color-specific search strategy would have required the simultaneous active maintenance of two task-relevant colors. Adopting a feature-unspecific singleton search mode in Experiment 1 and a feature-specific task set that included only the target color in Experiment 2 provided a lower-cost alternative. In both instances, search could be guided by a single target property, thereby reducing the demands on working memory maintenance and top-down attentional control. ${ }^{3}$ It has previously been suggested that working memory may not be involved at all in visual search tasks where the target-defining property remains constant across trials. Woodman, Luck, and Schall (2007) measured search performance during the delay period of a working memory task and found only minimal interference effects when the identity of search targets was kept constant. In contrast, search performance was impaired by the concurrent working memory task when target features varied randomly across trials. These findings were interpreted as evidence that working memory guides visual search when target identity changes frequently, whereas search for a fixed target may instead be based on an automatic retrieval of a task set from long-term memory. If this assumption is correct, the choice of a singleton search mode in Experiment 1 and of a target-color-specific task set in Experiment 2 would have enabled participants to perform visual search without any involvement of working memory.

Even though the present study demonstrated that attentional capture by irrelevant-color singleton cues is strongly affected by top-down search strategies, the ERP data obtained in Experiment 2 suggest that the top-down control of attentional selectivity by a feature-specific task set was not perfect. In this experiment, irrelevant-color and nontarget-color cues triggered reliable N2pc components, and subsequent analyses revealed that these components were present on trials with fast responses to same-location targets and slow responses to different-location targets, but not on same-location slow or different-location fast trials. This pattern of N2pc results suggests that irrelevantcolor and nontarget-color cues did capture attention on a subset of trials in Experiment 2, and may seem difficult to reconcile with the fact that across all trials, no behavioral spatial cuing effects were observed for these two types of cues. A possible explanation for this apparent discrepancy between behavioral and ERP results is that behavioral spatial cuing effects elicited on trials where attention was captured by irrelevant-color and nontargetcolor cues were compensated for by inverted spatial cuing effects (i.e., faster RTs on different-location than on samelocation trials) on trials without capture. Previous experiments where participants searched for color targets presented among heterogeneous color distractors and were thus forced into adopting a color-specific feature search mode (Eimer et al., 2009; Lamy et al., 2004) have indeed found inverted spatial cuing effects in response to taskirrelevant color singleton cues, which were interpreted as evidence for location-specific inhibition (see also Lamy
\& Egeth, 2003, for similar findings). The absence of behavioral spatial cuing effects for irrelevant-color and nontarget-color cues in Experiment 2 may thus be due to the fact that these cues captured attention on some trials but triggered location-specific inhibition on others, resulting in opposite behavioral effects that canceled each other out across all trials.

The hypothesis that the feature-specific task set adopted in Experiment 2 did not always prevent irrelevant-color cues from capturing attention also suggests that the effects of generalized top-down task sets on attentional capture, as observed in the present study, are gradual rather than absolute. The strong attenuation of the N2pc component to irrelevant-color cues as compared with target-color cues in Experiment 2 relative to Experiment 1 most likely occurred because irrelevant-color cues were much less likely to capture attention when a feature-specific task set was adopted than under a singleton search mode, but this does not imply that attentional capture was prevented on all trials. A potentially important factor that may determine the strength of this residual capture effect is the type of search array used. In the present study, targets were always color singletons, whereas in previous experiments that investigated feature-specific search modes, color targets were often nonsingletons presented among heterogeneous distractors (e.g., Eimer et al., 2009; Lamy et al., 2004). Under these conditions, inverted behavioral spatial cuing effects indicative of location-specific inhibition were reliably observed for irrelevant-color singleton cues; there was no evidence for an N2pc in response to these cues, suggesting that attentional capture was successfully prevented on virtually all trials. In contrast, results similar to those found in Experiment 2 (a small and delayed N2pc to irrelevant-color singleton cues in the absence of behavioral spatial cuing effects) were observed in another ERP experiment (Eimer \& Kiss, 2008, Experiment 1) where observers searched for onset targets. These observations suggest that the impact of feature-specific top-down task sets on attentional selectivity may be generally more pronounced when visual search targets are nonsingletons.

In summary, the present study provided behavioral as well as electrophysiological evidence for the existence of two generalized top-down search modes. The choice of a specific search strategy may be guided by the general aim to reduce or eliminate the involvement of working memory load in the control of visual search. When the singleton search mode is active, attentional capture will be triggered by task-relevant and task-irrelevant singletons. When a feature search mode is chosen, singletons that do not match the current task set are much less likely to capture attention.

\section{AUTHOR NOTE}

This research was supported by a grant from the Biotechnology and Biological Sciences Research Council (BBSRC), U.K. M.E. holds a Royal Society-Wolfson Research Merit Award. The authors thank Roberto Dell'Acqua, Clayton Hickey, Jeremy Wolfe, and an anonymous referee for comments. Correspondence concerning this article should be addressed to M. Eimer, Department of Psychology, Birkbeck College, University of London, Malte Street, London WC1E 7HX, England (e-mail: m.eimer@bbk.ac.uk). 


\section{REFERENCES}

Bacon, W. F., \& Egeth, H. (1994). Overriding stimulus-driven attentional capture. Perception \& Psychophysics, 55, 485-496.

EIMER, M. (1996). The N2pc component as an indicator of attentional selectivity. Electroencephalography \& Clinical Neurophysiology, 99, 225-234.

EImer, M., \& Kiss, M. (2008). Involuntary attentional capture is determined by task set: Evidence from event-related brain potentials. Journal of Cognitive Neuroscience, 20, 1423-1433.

Eimer, M., Kiss, M., Press, C., \& Sauter, D. (2009). The roles of feature-specific task set and bottom-up salience in attentional capture: An ERP study. Journal of Experimental Psychology: Human Perception \& Performance, 35, 1316-1328.

Folk, C. L., \& Remington, R. W. (1998). Selectivity in distraction by irrelevant featural singletons: Evidence for two forms of attentional capture. Journal of Experimental Psychology: Human Perception \& Performance, 24, 847-858.

FolK, C. L., \& Remington, R. W. (2008). Bottom-up priming of topdown attentional control settings. Visual Cognition, 16, 215-231.

Folk, C. L., Remington, R. W., \& Johnston, J. C. (1992). Involuntary covert orienting is contingent on attentional control settings. Journal of Experimental Psychology: Human Perception \& Performance, 18, 1030-1044.

Folk, C. L., Remington, R. W., \& Wright, J. H. (1994). The structure of attentional control: Contingent attentional capture by apparent motion, abrupt onset, and color. Journal of Experimental Psychology: Human Perception \& Performance, 20, 317-329.

GIRELLI, M., \& LUCK, S. J. (1997). Are the same attentional mechanisms used to detect visual search targets defined by color, orientation, and motion? Journal of Cognitive Neuroscience, 9, 238-253.

Hickey, C., McDonald, J. J., \& Theeuwes, J. (2006). Electrophysiological evidence of the capture of visual attention. Journal of Cognitive Neuroscience, 18, 604-613.

Lamy, D., \& Egeth, H. E. (2003). Attentional capture in singletondetection and feature-search modes. Journal of Experimental Psychology: Human Perception \& Performance, 29, 1003-1020.

LAmy, D., Leber, A., \& EgETH, H. E. (2004). Effects of task relevance and stimulus-driven salience within feature search mode. Journal of Experimental Psychology: Human Perception \& Performance, 30, 1019-1031.

Lien, M.-C., Ruthruff, E., Goodin, Z., \& Remington, R. W. (2008). Contingent attentional capture by top-down control settings: Converging evidence from event-related potentials. Journal of Experimental Psychology: Human Perception \& Performance, 34, 509-530.

LuCK, S. J., \& Hillyard, S. A. (1994a). Electrophysiological correlates of feature analysis during visual search. Psychophysiology, 31, 291308.

LuCK, S. J., \& Hillyard, S. A. (1994b). Spatial filtering during visual search: Evidence from human electrophysiology. Journal of Experimental Psychology: Human Perception \& Performance, 20, 10001014

Mazza, V., Turatto, M., Umiltà, C., \& Eimer, M. (2007). Attentional selection and identification of visual objects are reflected by distinct electrophysiological responses. Experimental Brain Research, 181, 531-536.

Miller, J., Patterson, T., \& Ulrich, R. (1998). Jackknife-based method for measuring LRP onset latency differences. Psychophysiology, 35, 99-115.

Perrin, F., Pernier, J., Bertrand, O., \& Echallier, J. (1989). Spherical splines for scalp potential and current density mapping. Electroencephalography \& Clinical Neurophysiology, 72, 184-187.

Theeuwes, J. (1991). Cross-dimensional perceptual selectivity. Perception \& Psychophysics, 50, 184-193.

Theeuwes, J. (1992). Perceptual selectivity for color and form. Perception \& Psychophysics, 51, 599-606.

Theeuwes, J. (2004). Top-down search strategies cannot override attentional capture. Psychonomic Bulletin \& Review, 11, 65-70.

Theeuwes, J., Atchley, P., \& Kramer, A. F. (2000). On the time course of top-down and bottom-up control of visual attention. In S. Monsell \& J. Driver (Eds.), Attention and performance XVIII: Control of cognitive processes (pp. 105-124). Cambridge, MA: MIT Press.

Woodman, G. F., \& LUCK, S. J. (1999). Electrophysiological measurement of rapid shifts of attention during visual search. Nature, 400, 867-869.

Woodman, G. F., LucK, S. J., \& SchaLL, J. D. (2007). The role of working memory representations in the control of attention. Cerebral Cortex, 17, 118-124.

Yantis, S., \& EgEth, H. (1999). On the distinction between visual salience and stimulus-driven attentional capture. Journal of Experimental Psychology: Human Perception \& Performance, 25, 661-676.

YANTIS, S., \& Jonides, J. (1984). Abrupt visual onsets and selective attention: Evidence from visual search. Journal of Experimental Psychology: Human Perception \& Performance, 10, 601-621.

\section{NOTES}

1. Abrupt onset items may be an exception to this general rule, since they appear to capture attention regardless of top-down task set (e.g., Lamy \& Egeth, 2003; Yantis \& Jonides, 1984).

2. For this analysis, behavioral and ERP data obtained on trials with irrelevant-color and nontarget-color singleton cues were combined, to ensure that the signal-to-noise ratio of ERPs remained sufficiently high after the RT median split.

3. It should be emphasized that this account of the role of working memory load for the selection between different search strategies cannot be directly demonstrated by the present experiments, since working memory load was not explicitly manipulated. In a future study, attentional capture by color singleton cues could be measured in a task where search arrays include one of three equally likely color singletons (two response-relevant target colors and one nontarget color). Because maintaining a feature-specific task set that includes both target colors but not the nontarget color will impose substantial demands on working memory, participants might opt for a lower cost singleton search mode under such conditions.

(Manuscript received October 20, 2009; revision accepted for publication January 10, 2010.) 